\title{
ACERCA DEL ESTATUS DE LA PSICOLOGÍA EMPÍRICA EN LA CRÍTICA DE LA RAZÓN PURA Y LOS PRIMEROS PRINCIPIOS METAFÍSICOS DE LA CIENCIA DE LA NATURALEZA DE KANT
}

\author{
ON THE STATUS OF EMPIRICAL PSYCHOLOGY IN KANT'S \\ CRITIQUE OF PURE REASON AND METAPHYSICAL FOUNDATIONS \\ OF NATURAL SCIENCE
}

\author{
Martín Arias-Albisu* \\ Universidad de Buenos Aires / CONICET - Argentina
}

Recibido septiembre de 2019/Received September, 2019

Aceptado mayo de 2020/Accepted May, 2020

\begin{abstract}
RESUMEN
Para Kant, la psicología empírica es la disciplina que estudia el sentido interno. En este sentido interno percibimos los estados de nuestra propia mente. En sus Primeros principios metafísicos de la ciencia de la naturaleza, Kant afirma que esta psicología empírica, a diferencia de la física y la química, no puede alcanzar el estatus de una ciencia de la naturaleza. El objetivo de este artículo es mostrar que las limitaciones en el potencial cognitivo de la psicología empírica presentadas en la obra mencionada son consecuencia del hecho de que esta disciplina se ocupa del sentido interno, así como este es expuesto, principalmente, en la Crítica de la razón pura. A diferencia de todos los trabajos de comentaristas acerca de este tema, este artículo combina, en un estudio único, un análisis detallado de las implicancias de la naturaleza del sentido interno y un examen cuidadoso de las limitaciones cognitivas de la psicología empírica llevado a cabo teniendo en cuenta nuestro análisis de tal naturaleza del sentido interno.

Palabras Clave: Kant, Psicología Empírica, Crítica de la Razón Pura, Primeros Principios Metafísicos de la Ciencia de la Naturaleza.
\end{abstract}

\begin{abstract}
According to Kant, empirical psychology is a discipline that studies inner sense. In this inner sense we perceive the states of our own mind. In his Metaphysical Foundations of Natural Science, Kant states that this empirical psychology, in contrast to physics and chemistry, cannot reach the status of a natural science. The aim of this article is to show that the limitations in the cognitive potential of empirical psychology presented in the aforementioned work are consequence of the fact that this discipline deals with inner sense, as it is expounded, mainly, in the Critique of Pure Reason. Unlike all scholarly work on the subject, this article combines, in a single study, a detailed analysis of the implications of the nature of inner sense and a thorough examination of the cognitive limitations of empirical psychology carried out taking into account our analysis of such nature of the inner sense.
\end{abstract}

Key Words: Kant, Empirical Psychology, Critique of Pure Reason, Metaphysical Foundations of Natural Science.

Según Kant, la psicología empírica es una disciplina que examina los fenómenos del sentido interno. Mediante este sentido percibimos nuestros estados internos, es decir, los estados de nuestra propia mente. Semejantes estados están sujetos a la forma del sentido interno, esto es, el tiempo. En el "Prólogo" a sus Primeros principios metafísicos de la ciencia de la naturaleza (Metaphysische Anfangsgründe der Naturwissenschaft = MAN), Kant afirma que la psicología empírica, a diferencia de la física y la química, no es una ciencia natural (AA IV, pp. 468-471) ${ }^{1}$. El objetivo principal de 
este artículo es mostrar que las limitaciones del potencial cognitivo de la psicología empírica, en virtud de las cuales esta psicología no puede ser una ciencia natural, se originan en la circunstancia de que el campo de estudio de esta disciplina es el sentido interno, así como este sentido es concebido y expuesto, principalmente, en la Crítica de la razón pura (Kritik der reinen Vernunft $=\mathrm{KrV}$ ).

Con el fin de conseguir nuestro objetivo fundamental, dividiremos la exposición de este artículo en tres secciones principales. En primer lugar, expondremos la doctrina del sentido interno de $\mathrm{KrV}$, poniendo especial énfasis en las características de este sentido que son relevantes para la comprensión de las limitaciones del potencial cognitivo de la psicología empírica presentadas en MAN. En segundo lugar, examinaremos las limitaciones en cuestión, y mostraremos que ellas se pueden comprender cabalmente a partir de la doctrina del sentido interno de $\mathrm{KrV}$ expuesta en la sección anterior. En tercer lugar, comentaremos sucintamente dos trabajos recientes dedicados a la temática de la psicología empírica en Kant (Frierson, 2014; Kraus, 2018) y señalaremos la importancia que tiene la comprensión de la doctrina kantiana del sentido interno para la evaluación de los dos trabajos mencionados.

Adelantamos que la característica más importante del sentido interno (como es concebido en la primera $C$ rítica $)^{2}$, que limita el potencial cognitivo de la psicología empírica, consiste en que en el mismo no hay nada permanente que permita la aplicación de las categorías de la relación (substancia, causalidad y comunidad). El aporte principal de nuestro trabajo es la combinación, en un solo estudio, de, por un lado, un análisis detallado de las implicaciones de la ausencia de algo permanente en el sentido interno y, por el otro, un examen de las limitaciones cognitivas de la psicología empírica efectuado a la luz de estas implicaciones. Ninguno de los trabajos mencionados en este artículo presenta esta combinación ${ }^{3}$. Haremos referencia, mayormente en notas al pie, a estos diversos estudios al tratar diferentes cuestiones específicas en el desarrollo de nuestra exposición.

\section{LA DOCTRINA DEL SENTIDO INTERNO SEGÚN LA CRÍTICA DE LA RAZÓN PURA}

Abordemos entonces la temática del sentido interno. Según Kant, en este sentido nos es dado un flujo incesante de fenómenos en el que no se encuentra nada permanente. Por este motivo, en el ámbito de los fenómenos internos no es posible en sentido estricto la aplicación de las categorías, especialmente de las tres categorías de la relación, a saber, las de substancia, causalidad y comunidad ${ }^{4}$. Consideremos en primer lugar la categoría de substancia. Esta categoría solo puede aplicarse a un fenómeno permanente, y los fenómenos de esta clase se encuentran exclusivamente en el espacio. Como es sabido, el espacio no es una forma del sentido interno, sino solamente del sentido externo, en el que son dados los fenómenos diferentes de nosotros mismos ${ }^{5}$. Por tanto, la categoría de substancia no puede aplicarse estrictamente hablando a los fenómenos internos (A22/B37ss., A144/B183, A182-184/B224-227, B275-276 y B291). En segundo lugar, debe notarse que tampoco las categorías de causalidad y comunidad pueden aplicarse en sentido riguroso a los fenómenos internos, porque su aplicación presupone la aplicación de la categoría de substancia.

El hecho de que las categorías, cuando menos las de la relación, no puedan aplicarse estrictamente hablando a los fenómenos internos tiene como consecuencia una cierta falta de objetividad de estos fenómenos. Esta falta de objetividad consiste, principalmente, en la imposibilidad de constituir, en el dominio del sentido interno, la experiencia de una comunidad causalmente interrelacionada de objetos substanciales relativamente permanentes ${ }^{6}$ dotados de estados o accidentes sucesivos ${ }^{7}$.

Según Kant, un acontecimiento es un cambio de estado de una substancia. La experiencia de un acontecimiento presupone no solo la aplicación de la categoría de substancia, sino también, especialmente, la de la categoría de causalidad. Como las categorías de la relación no pueden aplicarse en sentido estricto en el ámbito del sentido interno, no es posible tener una experiencia de un acontecimiento en el ámbito en cuestión. Por este motivo, el flujo constante de fenómenos internos no puede estar compuesto por cambios de estado, esto es, por acontecimientos. Esta imposibilidad entraña la imposibilidad de establecer, en sentido estricto, relaciones causales entre fenómenos internos. Efectivamente, estas relaciones se establecen propiamente hablando entre estados de substancias (por ejemplo, el contacto entre un libro y una lámpara) y acontecimientos (por ejemplo, el reemplazo del estado de la lámpara en tanto erguida sobre un escritorio por el estado de la misma en tanto caída sobre este escritorio). Asimismo, la 
imposibilidad de establecer, estrictamente hablando, esas relaciones implica la imposibilidad de formular, en sentido riguroso, leyes causales que se refieran a esas relaciones y las expliquen.

Intentaremos exponer más detalladamente la concepción kantiana del acontecimiento ${ }^{8}$. Los acontecimientos son constituidos en tanto tales en virtud de, particularmente, la aplicación de la categoría de causalidad. La constitución de un acontecimiento entraña la remisión de este, en tanto efecto, a un estado precedente, en tanto causa, del que se seguiría en conformidad con una ley empírica. Sin embargo, la aplicación de la categoría de causalidad no permite por sí misma establecer cuál sea ese estado precedente y cuál sea esa ley empírica. Más bien, mediante esa aplicación estos dos últimos términos quedan indeterminados. Si el acontecimiento experimentado fuese la caída de una lámpara sobre un escritorio, la ley empírica indeterminada tendría la forma siguiente: "siempre que se da un estado $x$, todas las lámparas se caen". Una investigación empírica ulterior podría determinar el estado $x$ como, por ejemplo, el contacto entre ciertos libros y lámparas erguidas sobre escritorios. En este caso, la ley empírica determinada sería: "siempre que hay cierto contacto entre libros y lámparas, todas las lámparas se caen" (cf. A144/ B183, A189/B232ss y Longuenesse, 2000, pp. 363370). Es preciso destacar que esta ley empírica podría ser explicada por otra ley empírica más general con respecto a la cual la primera sería un caso particular ${ }^{9}$. Además, debe recordarse que la aplicación de la categoría de causalidad presupone la de la categoría de substancia ${ }^{10}$.

La experiencia de un acontecimiento nos permite distinguir nítidamente entre los dos estados de una substancia en cuya sucesión consiste el mismo. En muchos casos es posible examinar detenidamente el estado inicial y el estado final de dicha substancia. En nuestro ejemplo, el estado inicial es el estado de la lámpara en tanto erguida sobre un escritorio, y el estado final es el estado de esta lámpara en tanto caída sobre este escritorio. Más aún, en el caso de objetos manipulables como las lámparas y los libros, podemos efectuar experimentos repetibles con el fin de comprobar si estas lámparas se caen cuando hay cierto contacto entre ellas y determinados libros, es decir, si ese contacto es la causa de esa caída.

La exposición precedente permite sostener la tesis según la cual el flujo de fenómenos internos puede entenderse, cuando mucho, como un análogo de un acontecimiento en el ámbito de los fenómenos externos. Hay semejanza entre estos dos casos solamente porque en ambos se encuentra una sucesión de representaciones. Sin embargo, existen grandes diferencias entre ellos. En el caso de la experiencia de un acontecimiento en el ámbito de los fenómenos externos, podemos hacer experimentos y observaciones rigurosas que permiten mostrar que nuestras representaciones sucesivas hacen referencia a un acontecimiento producido por una causa determinada. En efecto, en principio es posible identificar en la experiencia externa estos términos, a saber, el estado inicial, el estado final y la causa del cambio de estado. Por el contrario, en el caso de los fenómenos internos, nos es dado un flujo constante que ni siquiera nos permite distinguir nítidamente entre dos representaciones sucesivas ${ }^{11}$. Tan solo podemos, efectuando una analogía con los acontecimientos externos, intentar distinguir entre estas representaciones. Esta distinción es una condición de posibilidad del intento de explicar una determinación de una representación como consecuencia de otra determinación de una representación. En esta última operación, efectuamos una analogía con las relaciones causales entre fenómenos externos ${ }^{12}$.

Citamos a continuación un pasaje de $\mathrm{KrV}$ que provee sustento para las afirmaciones presentadas en esta sección:

para entender la posibilidad de las cosas como consecuencia de las categorías, y por consiguiente, para exponer la realidad objetiva de las últimas, no solo necesitamos intuiciones, sino incluso siempre intuiciones externas (...). Para exhibir la alteración como la intuición correspondiente al concepto de causalidad, debemos tomar como ejemplo el movimiento, como alteración en el espacio (...). Alteración es enlace de determinaciones opuestas entre sí de manera contradictoria, en la existencia de una y la misma cosa. Cómo es posible que de un estado se siga otro de la misma cosa, opuesto a él, [es algo que] (...) [Autor: solo puede hacerse inteligible por la intuición] del movimiento de un punto en el espacio, cuya existencia en diferentes lugares (como consecuencia de determinaciones contrapuestas) es lo que, primeramente, nos hace intuitiva la alteración. Pues para hacernos pensables luego las alteraciones internas mismas, debemos hacernos concebible 
figurativamente el tiempo, como forma del sentido interno, mediante una línea, y la alteración interna, mediante el trazado de esa línea (movimiento); y por tanto, [debemos hacernos concebible] la existencia sucesiva de nosotros mismos en diferentes estados, mediante la intuición externa; el fundamento propio de todo ello es este: que toda alteración presupone algo permanente en la intuición, aun ya solo para ser percibida como alteración; pero en el sentido interno no se encuentra ninguna intuición permanente (B291-292).

Ofreceremos únicamente tres indicaciones respecto de este pasaje. En primer lugar, el concepto de realidad objetiva de las categorías hace referencia a que hay una relación efectiva entre estas y los objetos empíricos. Como es sabido, en la filosofía trascendental kantiana esta relación es consecuencia de la aplicación de las categorías a intuiciones empíricas, la que constituye a estas intuiciones como objetos empíricos en sentido estricto.

En segundo lugar, el concepto de alteración (Veränderung) se refiere al concepto de acontecimiento. Debido a que el ejemplo de acontecimiento dado por Kant, esto es, el movimiento de un punto en el espacio, se refiere a una intuición correspondiente a un concepto matemático, y no a objetos empíri$\cos$, que son los que aquí nos interesan, retomamos nuestro ejemplo de la caída de una lámpara sobre un escritorio con el fin de explicar la terminología kantiana relativa a los acontecimientos. Según esta terminología, tal acontecimiento puede describirse como un cambio (Wechsel) de accidentes (cambio del estado de la lámpara en tanto de pie sobre el escritorio por el estado de la misma en tanto caída sobre este escritorio) y como una alteración (Veränderung) del objeto substancial que permanece (la lámpara) ${ }^{13}$.

En tercer lugar, en el texto citado se pone de manifiesto que la categoría de causalidad solo puede aplicarse en sentido riguroso a intuiciones empíricas en el espacio que, en virtud de esta aplicación, así como la de las restantes categorías, se constituyen como objetos empíricos en el sentido estricto del término. La contribución de la categoría de causalidad a la conformación de la objetividad consiste en la constitución de una experiencia de acontecimientos. Estos acontecimientos posibilitados por esta aplicación solo pueden encontrarse en el espacio, porque la experiencia de ellos presupone la presencia de algo permanente dado en la intuición externa (véase supra, n. 13). En lo que hace a "las alteraciones internas", únicamente podemos "hacernos pensables" las mismas al intentar comprenderlas en términos de las alteraciones externas (Ibíd.). Hemos explicado anteriormente, en la presente sección, las diferencias entre las alteraciones espaciales y las cuasialteraciones del sentido interno.

En síntesis, ya que, como adelantamos, no es posible establecer relaciones causales en sentido estricto entre fenómenos internos, las leyes que se refieren a estas relaciones ${ }^{14}$ no pueden ser leyes causales en el sentido riguroso del término ${ }^{15}$.

Antes de terminar esta sección, afrontaremos una posible objeción a nuestra interpretación según la cual las categorías, especialmente las de la relación, no pueden aplicarse a los fenómenos internos. Esta objeción podría formularse a partir de las tesis expuestas por Kant en el capítulo "Del esquematismo de los conceptos puros del entendimiento" de su KrV (A137/B176ss.), según las cuales parecería que las categorías tienen que determinar el tiempo mismo.

En el capítulo mencionado, Kant afirma que las categorías solo pueden aplicarse a las intuiciones empíricas si se establece una relación de homogeneidad entre las primeras y las segundas. Esta relación de homogeneidad se produce mediante las representaciones mediadoras denominadas "esquemas trascendentales" (A137-139/B176-178). Según Kant, los esquemas trascendentales deben entenderse como "determinaciones trascendentales del tiempo" (transzendentale Zeitbestimmungen. A138-139/B177-178 y A145/B184). Esta afirmación puede comprenderse en el sentido de que los esquemas mencionados son el resultado de la conformación del tiempo por parte de las diferentes categorías. Las intuiciones empíricas adoptarían estas conformaciones en tanto que estén dadas en el sentido interno. De esta manera, estas intuiciones presentarían una cierta organización que guarda correspondencia con las categorías, con lo que se establecería una relación de homogeneidad entre ambas instancias. En consecuencia, las categorías podrían aplicarse a las intuiciones empíricas, y estas últimas serían constituidas como una experiencia de objetos empíricos en el sentido estricto del término ${ }^{16}$.

Lo que nos interesa destacar aquí es que, si la interpretación esbozada es correcta, entonces habría un sentido en el que las categorías determinan el tiempo. No se trata de una aplicación, en el sentido riguroso del término, al flujo de representaciones dado en el tiempo, contra cuya posibilidad 
hemos argumentado, sino de una determinación del tiempo mismo como forma sensible universal de los fenómenos. Sin embargo, debido a que nuestra argumentación, en parte, pretende establecer que las categorías no pueden aplicarse en ausencia de intuiciones espaciales, esta argumentación también parece estar en contradicción con la posibilidad de una determinación, por parte de las categorías, del tiempo mismo. Intentaremos entonces, a continuación, mostrar que puede sostenerse que, para Kant, las categorías no efectúan esa determinación. En efecto, se debe señalar que la interpretación, delineadas más arriba, de la caracterización kantiana de los esquemas trascendentales como determinaciones trascendentales del tiempo no es la única posible. Efectivamente, Zeitbestimmung no solo puede entenderse como determinación del tiempo, sino también como determinación temporal de, por ejemplo, lo empírico ${ }^{17}$. En este caso, los esquemas trascendentales serían conformaciones temporales de las intuiciones empíricas espacio-temporales. Estas conformaciones serían generadas por las distintas categorías. Al ser conformadas de esta manera, las intuiciones empíricas espacio-temporales se tornarían homogéneas con las categorías, con lo que las segundas podrían aplicarse a las primeras. De este modo, esas intuiciones empíricas serían constituidas como objetos empíricos en el sentido riguroso del término.

Para nuestros fines basta con haber señalado que no es necesario entender que, para Kant, las categorías tienen que determinar el tiempo mismo. Notemos además, en primer lugar, que varios reconocidos comentaristas han sostenido interpretaciones de los esquemas trascendentales compatibles con la lectura de Zeitbestimmung como determinación tempora ${ }^{18}$. En segundo lugar, se debe destacar que esta lectura es compatible con las descripciones que ofrece Kant de los diferentes esquemas trascendentales. Tomemos como ejemplo el esquema trascendental correspondiente a la categoría de substancia. Este esquema es "la permanencia de lo real en el tiempo, es decir, la representación de ello como substrato de la determinación empírica general del tiempo, [substrato] que permanece, pues, mientras todo lo demás cambia" (A144/B183). En virtud de la aplicación de la categoría de substancia, por intermedio de este esquema trascendental, las intuiciones empíricas espacio-temporales adoptan una cierta conformación temporal, a saber, la que consiste en la relación entre un substrato permanente y accidentes mudables de este substrato. Es claro que esta conformación no es una determinación del tiempo puro, sino una determinación temporal de lo empírico. En efecto, esta conformación consiste en la relación entre lo real empírico permanente y lo real empírico mudable (cf. supra, n. 6) ${ }^{19}$.

\section{LAS LIMITACIONES DEL POTENCIAL COGNITIVO DE LA PSICOLOGÍA EMPÍRICA SEGÚN MAN}

El objetivo de la presente sección es estudiar las limitaciones del potencial cognitivo de la psicología empírica que resultan de la ausencia de algo permanente en el sentido interno. Consideremos en primer lugar el "Prólogo" a MAN. En este texto, entre otras cosas, Kant muestra que la psicología empírica, a diferencia de la física matemática, no es una ciencia natural en un sentido propio del término, porque no es posible aplicar adecuadamente la matemática a los fenómenos internos (MAN, AA IV, p. 471). La aplicación adecuada de la matemática es legitimada por la parte metafísica de la física matemática, parte metafísica que es el fundamento de la parte empírica de la física matemática y es expuesta en MAN. Esta parte metafísica toma al movimiento como característica principal de la materia y se refiere exclusivamente a los objetos materiales espaciales (MAN, AA IV, pp. 476-477). Ahora bien, según $\mathrm{KrV}$, los fenómenos internos no están sometidos como los fenómenos externos a la forma del espacio, sino únicamente a la del tiempo. Por tanto, la teoría del sentido interno de $\mathrm{KrV}$ explica el hecho de que la disciplina que estudia a este sentido, a saber, la psicología empírica, no sea una ciencia natural en sentido propio.

Efectivamente, en el "Prólogo" a MAN, Kant escribe:

[p]ero la doctrina empírica del alma tiene que permanecer siempre más alejada todavía del rango de una así llamada ciencia de la naturaleza en sentido propio que la química misma; primeramente, porque la matemática no es aplicable a los fenómenos del sentido interno ni a sus leyes, a menos que se quiera tomar en cuenta, solamente, la ley de continuidad en el flujo de las alteraciones internas del mismo sentido; pero esto sería una extensión del conocimiento que se comportaría, respecto de aquella que la matemática proporciona a la doctrina del cuerpo, más o menos como la doctrina de las propiedades de la línea 
recta respecto de toda la geometría. Pues la intuición interna pura en la cual los fenómenos del alma deben construirse es el tiempo, que tiene solamente una dimensión (MAN, AA IV, p. 471).

Un análisis detallado del pasaje citado debería incluir un examen del sentido en el cual el campo de estudio de la física matemática admite una matematización adecuada. Este examen excede los límites de nuestro trabajo ${ }^{20}$. Para nuestros fines, basta la breve explicación acerca de esta cuestión ofrecida en el párrafo anterior a las oraciones citadas. Esta explicación, como se vio, vincula la imposibilidad de matematizar adecuadamente el campo de estudio de la psicología empírica con la doctrina del sentido interno de $\mathrm{KrV}$. Como se afirma en el pasaje citado, la imposibilidad en cuestión está conectada con el hecho de que el sentido interno posee solo una dimensión, a saber, la temporal.

En el "Prólogo" a MAN, Kant también da a entender que la psicología empírica no puede ser una ciencia en un sentido impropio del término como la química flogística ${ }^{21}$ (MAN, AA IV, p. 471). En un trabajo anterior hemos intentado mostrar que esta química es una ciencia, aunque en sentido impropio, porque puede ser sistemática y experimental (Arias-Albisu, 2017). De hecho, la psicología empírica no puede ser una ciencia en sentido impropio, fundamentalmente, porque no es posible realizar experimentos ni en el sentido interno propio ni en el ajeno (MAN, AA IV, p. 471).

Se debe señalar que, a diferencia de la física matemática, que contiene una parte metafísica que fundamenta la aplicación adecuada de la matemática dentro de su dominio, la química flogística y la psicología empírica están justificadas exclusivamente de manera empírica. La diferencia fundamental entre estas dos últimas disciplinas consiste en que, mientras que las leyes de la química flogística se vuelven más probables en virtud de la realización exitosa de experimentos materiales y repetibles, el sustento empírico de la psicología empírica consiste en un flujo constante de fenómenos internos en el que la ausencia de algo permanente hace imposible la experiencia de objetos substanciales y, por tanto, la realización de exámenes observacionales y experimentos materiales y repetibles (MAN, AA IV, p. 471). Como señalamos más arriba, las leyes que versan acerca de fenómenos internos no pueden ser en sentido estricto leyes causales referidas a relaciones causales. Más bien, como adelantamos, estas cuasileyes se refieren a vínculos tentativos entre dos clases de determinaciones de representaciones; vínculos que, propiamente hablando, no pueden ponerse rigurosamente a prueba ${ }^{22}$.

A continuación del pasaje en el que Kant sustenta la tesis de que la psicología empírica no es una ciencia en sentido propio como la física matemática, se encuentra un pasaje en el que nuestro autor expone el carácter no experimental de la psicología empírica. Este señalamiento tiene por objetivo mostrar que la psicología empírica no es una ciencia en sentido impropio como la química. Citamos este pasaje a continuación:

[p]ero la doctrina empírica del alma tampoco puede acercarse jamás a la química como arte sistemático de análisis o doctrina experimental, porque en ella lo múltiple de la observación interna solo se puede separar mediante una mera división en el pensamiento, pero no puede mantenerse separado y conectarse de nuevo a voluntad; pero menos aún puede someterse a otro sujeto pensante a experimentos convenientes a nuestro propósito, e incluso la observación en sí ya altera y desplaza el estado del objeto observado. Por eso, la doctrina empírica del alma nunca puede llegar a ser algo más que una doctrina histórica natural del sentido interno y, como tal, tan sistemática como sea posible, es decir, una descripción natural del alma, pero no una ciencia del alma, ni siquiera una doctrina psicológica experimental (MAN, AA IV, p. 471).

Efectuaremos tres señalamientos respecto del texto citado. En primer lugar, Kant afirma: "lo múltiple de la observación interna solo se puede separar mediante una mera división en el pensamiento, pero no puede mantenerse separado y conectarse de nuevo a voluntad". Esta tesis de Kant hace referencia a la ya mencionada imposibilidad de efectuar, en el dominio del sentido interno propio, especialmente, experimentos. No es posible realizar experimentos en el dominio en cuestión porque las partes constitutivas de los fenómenos internos no pueden ser manipuladas como sí pueden serlo las de los fenómenos materiales externos. Esta imposibilidad, como ya también indicamos, explica el hecho de que la psicología empírica no pueda ser una ciencia natural en un sentido impropio del 
término como la química flogística. En cambio, esta disciplina puede ser únicamente una sistemática "descripción natural del alma".

En segundo lugar, es evidente que, como también se da a entender en el pasaje citado, la imposibilidad de realizar experimentos materiales y repetibles no se refiere solamente al ámbito del sentido interno nuestro, sino también al del sentido interno de sujetos diferentes de nosotros. Efectivamente, Kant escribe: "pero menos aún puede someterse a otro sujeto pensante a experimentos convenientes a nuestro propósito". Esta imposibilidad se origina en el hecho de que el sentido interno nos permite solamente percibir los estados de la propia mente.

Lo que quisiéramos subrayar es que la circunstancia, según la cual la imposibilidad de realizar experimentos en el sentido interno propio y en el ajeno pueda entenderse a partir de la exposición precedente de temáticas de $\mathrm{KrV}$, sustenta nuestra tesis de que la comprensión de las indicaciones acerca del escaso potencial cognitivo de la psicología empírica presentadas en el "Prólogo" a MAN presupone la comprensión acabada de la doctrina del sentido interno de $\mathrm{KrV}$.

En tercer lugar, nos limitamos a señalar que en el citado pasaje del "Prólogo" a MAN se presenta una deficiencia en el potencial cognitivo de la psicología empírica que no puede inferirse a partir de la exposición de KrV. Esta deficiencia consiste en que la observación de determinados fenómenos internos puede modificar a estos últimos ${ }^{23}$. El examen de esta deficiencia no es relevante para los fines del presente trabajo ${ }^{24}$.

\section{BREVE REVISIÓN DE LAS INTERPRETACIONES DE FRIERSON Y KRAUS}

La exposición llevada a cabo hasta aquí nos permitirá hacer, en esta sección, algunos señalamientos acerca de dos trabajos recientes que se ocupan de la concepción kantiana de la psicología empírica. Elegimos estos dos trabajos porque, como mostraremos, ponen en evidencia la necesidad imperiosa de tener en cuenta, a la hora de abordar el problema del estatus de la psicología empírica, la temática de la ausencia de algo permanente en el sentido interno tratada principalmente en la Crítica de la razón pura.

En primer lugar, Partrick R. Frierson (2014) sostiene que, para Kant, la psicología empírica, al igual que la química flogística, es una ciencia natural, aunque en un sentido impropio del término (p. 26). Según Frierson "los objetos empíricos son substancias que sufren alteraciones en virtud de poderes causales que operan de acuerdo con leyes naturales (véase B224ss.). Para los fenómenos del sentido interno en particular, esto hace posible una psicología empírica que estudia los poderes del alma humana de acuerdo con las leyes naturales de su operación" (p. 5). Esta psicología empírica es sistemática, y también es "mecanicista en el sentido amplio de que explica estados mentales particulares apelando a causas eficientes ocasionantes que provocan efectos de acuerdo con la operación de poderes caracterizados por leyes de la naturaleza" (p. 49).

Frierson tiene en cuenta los pasajes de MAN comentados en la segunda sección de este artículo. Sin embargo, tras examinar estos pasajes y considerar los aportes de otros comentaristas, Frierson concluye que, para Kant, si bien la psicología empírica no puede ser una ciencia natural en un sentido propio como la física, sí puede ser una ciencia natural en sentido impropio como la química. Según Frierson,

[1]a defensa limitada de Kant de la psicología empírica como una doctrina natural sistemática, pero no ciencia estricta, tiene tres importantes implicancias. En primer lugar, dado que la psicología empírica puede y tiene que operar dentro de su metafísica general de las categorías, en la cual la causalidad es la causalidad de los poderes de substancias, la psicología estudiará los poderes fundamentales del alma y las leyes que gobiernan la operación de esos poderes fundamentales. En segundo lugar, a diferencia de la física, no hay psicología a priori más allá de las meras condiciones de posibilidad de objetos, de modo que las únicas afirmaciones que la psicología puede hacer son aquellas que están basadas en la generalización empírica. En tercer lugar, como consecuencia del segundo punto, las leyes naturales investigadas por la psicología empírica pueden tener solo universalidad empírica, no la necesidad y universalidad estricta de las leyes físicas fundamentales (según la explicación de Kant de tales leyes). (p. 27).

En pocas palabras, las limitaciones de la psicología empírica que Kant habría querido destacar en los pasajes mencionados de MAN serían, 
fundamentalmente, que esta disciplina no tiene un contenido a priori específico y que, por tanto, sus afirmaciones no están basadas sino en la generalización empírica (pp. 26-27); como consecuencia de lo anterior, las leyes naturales de la psicología empírica no pueden alcanzar universalidad estricta, sino solamente empírica.

Llama la atención que en ninguna de las casi 300 páginas de su valioso libro Frierson examine los pasajes de Kant en los que se indica que la aplicación, en sentido estricto, de la categoría de substancia y, por consiguiente, de las categorías de causalidad y comunidad, requiere la presencia de algo permanente en el espacio ${ }^{25}$. En virtud de esta circunstancia, este documentado comentarista no alcanzó a percibir la diferencia entre los estatus de la química flogística y la psicología empírica (véase la sección anterior de este artículo).

Más prometedora parece la interpretación de Katharina T. Kraus (2018). Para Kraus, la psicología empírica "manifiesta un grado de sistematicidad suficiente para calificar como una ciencia natural impropia autocontenida, que concierne fundamentalmente a la vida mental humana más bien que a la acción humana" (p. 78). En una primera aproximación, la función que cumplen las substancias permanentes en el conocimiento de los fenómenos externos la cumple la idea regulativa del alma en el conocimiento de los fenómenos internos. Esta idea regulativa es presentada en la segunda parte del "Apéndice a la dialéctica trascendental" de KrV. Tras citar un pasaje de A672/B700, Kraus afirma: "la experiencia interna debería ser considerada como el conocimiento de estados mentales que inhieren en una substancia empírica; sin embargo, esta substancia empírica es solo presupuesta problemáticamente como la suma total de todos los fenómenos internos mediante una idea regulativa (...), más bien que como siendo afirmada sobre la base de la sensación" (p. 82).

$\mathrm{Al}$ comentar otro pasaje de la mencionada segunda parte del "Apéndice a la dialéctica trascendental", en donde también se expone la función regulativa de la idea de alma (A682-683/B710-711), Kraus sostiene: "buscamos explicaciones causales de fenómenos internos en términos de 'poderes' mentales causales y sus cambiantes estados mentales 'considerando todas las determinaciones como en un sujeto' dotado de tales poderes. Para dar un ejemplo, una persona puede conocer que su percepción de un animal salvaje causa su sentimiento de miedo solo al considerar la percepción y el sentimiento como 'estados [mentales] de uno y el mismo ser persistente"' (p. 82) ${ }^{26}$. Kraus agrega que la idea regulativa del alma permite conocer sistemáticamente no solo los predicados mentales, sino también, por ejemplo, las leyes psicológicas (p. 82).

Por último, Kraus mantiene que, aunque no sea posible someter a prueba empíricamente las leyes de la psicología empírica mediante "observaciones reproducibles", la función sistematizadora de la idea regulativa del alma posibilita que, "si los psicólogos pueden mostrar que una ley psicológica que han encontrado sobre la base de (auto-) observaciones empíricas encaja en el sistema de las leyes psicológicas, ellos tienen buenas razones para creer que esta ley 'se aproxima' a la validez universal" (p. 84).

Quisiéramos señalar que la discusión de Kraus pone de manifiesto la necesidad imperiosa de que haya algo permanente en el ámbito de los fenómenos internos con el fin de que sean posibles las explicaciones causales en el ámbito de la psicología empírica. Como afirma la autora, si no pudiésemos adscribir al mismo sujeto permanente una percepción de algo amenazador y un sentimiento de temor, sería imposible intentar explicar el segundo como ocasionado por la primera. La función regulativa de la idea de alma consiste, a grandes rasgos, en el postular esta entidad permanente como supuesto necesario para realizar explicaciones como la mencionada, aunque la existencia de esta entidad no pueda demostrarse. Nos limitamos a subrayar este punto, debido a que no es el objetivo de este artículo evaluar el intento de Kraus de resolver los problemas que ocasiona esa ausencia de algo permanente mediante la introducción de la función regulativa de la idea del alma. Asimismo, también la interpretación de Frierson muestra que toda valoración del estatus de la psicología empírica en Kant debe tener en cuenta la temática, expuesta en $\mathrm{KrV}$, de la imposibilidad de aplicar en sentido riguroso las categorías de la relación a los fenómenos internos. En el caso de este comentador, es evidente que su concepción de una psicología empírica como ciencia mecanicista debería haber concedido un espacio importante a la temática mencionada.

\section{CONCLUSIONES}

Tomando como base la doctrina del sentido interno expuesta en $\mathrm{KrV}$, especialmente en lo que hace a una cierta falta de objetividad de los 
fenómenos dados en dicho sentido, Kant presenta en MAN indicaciones acerca del escaso potencial cognitivo de la psicología empírica. Nuestro trabajo ha intentado mostrar que un examen del estatus del sentido interno es una condición, tal vez pueda decirse necesaria, de la comprensión de la mayoría de las mencionadas indicaciones. Esta tesis fue sustentada mediante una argumentación propia y una consideración de dos recientes trabajos de comentaristas. 


\section{Referencias}

Allison, H. E. (1992). El idealismo trascendental de Kant: una interpretación y defensa. Barcelona: Anthropos.

Allison, H. E. (2008). "Whatever begins to be must have a cause of existence": Hume's Analysis and Kant's Response. Philosophy and Phenomenological Research, 76(3), 525-546. https://doi. org/10.1111/j.1933-1592.2008.00156.x

Arias-Albisu, M. (2011). La concepción objetiva de la substancia en la Crítica de la razón pura de Kant. Estudios de Filosofía (Medellín), 44, 39-60.

Arias-Albisu, M. (2015). Las prescripciones metodológicas de la función regulativa de la razón teórica en la "Crítica de la razón pura” de Kant. Kant e-Prints, 10(1), 64-93.

Arias-Albisu, M. (2017). The Methodological Prescriptions of the "Appendix to the Transcendental Dialectic" of Kant's Critique of Pure Reason and the Foundations of Improper Science. Studia Kantiana, 15(2), 5-26.

Beck, L. W. (1978). A Prussian Hume and a Scottish Kant. En L. W. Beck, Essays on Kant and Hume (pp. 111-129). New Haven: Yale University Press.

Blanc-Brude, G. (2011). L'Anthropologie du point de vue pragmatique est-elle une psychologie? En S. Grapotte, M. Lequan, \& M. Ruffing (Eds.), Kant et les sciences (pp. 321-329). Paris: Vrin.

Blomme, H. (2011). Pourquoi la chimie ne peut-elle aspirer au titre de science proprement dite? En S. Grapotte, M. Lequan, \& M. Ruffing (Eds.), Kant et les sciences (pp. 159-168). Paris: Vrin.

Blomme, H. (2015). Kant's Conception of Chemistry in the Danziger Physik. En R. R. Clewis (Ed.), Reading Kant's Lectures (pp. 484-502). Berlin: De Gruyter.

Caimi, M. (2012). The logical structure of time according to the chapter on the Schematism. Kant Studien, 103(4), 415-428. DOI: https://doi.org/10.1515/kant-2012-0031.

Caimi, M. (2015). Der Schematismus der reinen Verstandesbegriffe. En R. Enskat (Ed.), Kants Theorie der Erfahrung (pp. 201-237). Berlín: De Gruyter.

Carrier, M. (2001). Kant's Theory of Matter and His Views on Chemistry. En E. Watkins (Ed.), Kant and the Sciences (pp. 205230). Oxford: Oxford University Press.

Düsing, K. (1995). Schema und Einbildungskraft in Kants Kritik der reinen Vernunft. En L. Kreimendahl (Ed.), Aufklärung und Skepsis. Studien zur Philosophie und Geistesgeschichte des 17. und 18. Jahrhunderts. Günter Gawlick zum 65. Geburtstag (pp. 47-71). Stuttgart-Bad Cannstatt: Frommann-Holzboog.

Friedman, M. (1992). Kant and the Exact Sciences. Cambridge: Harvard University Press.

Friedman, M. (2013). Kant's Construction of Nature. A reading of the Metaphysical Foundations of Natural Science. Cambridge: Cambridge University Press.

Frierson, P. R. (2014). Kant's Empirical Psychology. Cambridge: Cambridge University Press.

Gouaux, C. (1972). Kant's View on the Nature of Empirical Psychology. Journal of the History of the Behavioral Sciences, 8(2), 237-242.
Hatfield, G. (1992). Empirical, Rational, and Transcendental Psychology: Psychology as a Science and as Philosophy. En P. Guyer (Ed.), The Cambridge Companion to Kant (pp. 200-227). Cambridge: Cambridge University Press.

Hinske, N. (1999). Wolffs empirische Psychologie und Kants pragmatische Anthropologie: Zur Diskussion über die Anfänge der Anthropologie im 18. Jahrhundert. Aufklärung, 11(1), 97107. https://www.jstor.org/stable/24361420

Kant, I. (1900ss.). Kant's gesammelte Schriften. Deutsche (anteriormente Königlich Preußische) Akademie der Wissenschaften (Ed.). Berlin y otros: Walter de Gruyter y predecesores. $($ Akademie-Ausgabe $=\mathrm{AA})$.

Kant, I. (1989). Primeros principios metafísicos de la ciencia de la naturaleza. Madrid: Alianza. [MAN].

Kant, I. (1993). Primeros principios metafísicos de la ciencia de la naturaleza. México D. F.: Universidad Nacional Autónoma de México. [MAN].

Kant, I. (1998). Critique of Pure Reason. Cambridge: Cambridge University Press.

Kant, I. (2009a). Crítica de la razón pura. México D. F.: Fondo de Cultura Económica.

Kant, I. (2009b). Antropología en sentido pragmático. Buenos Aires: Losada.

Kraus, K. T. (2018). The Soul as the 'Guiding Idea' of Psychology: Kant on Scientific Psychology, Systematicity, and the Idea of the Soul. Studies in History and Philosophy of Science, 71, 77-88. https://doi.org/10.1016/j.shpsa.2017.11.010

Leary, D. E. (1982). Immanuel Kant and the Development of Modern Psychology. En W. R. Woodward, \& M. G. Asch (Eds.), The Problematic Science. Psychology in Nineteenth-Century Thought (pp. 17-42). New York: Praeger.

Leppäkoski, M. (1995). The Transcendental Schemata. En H. Robinson (Ed.), Proceedings of the Eight International Kant Congress (t. II, 1, pp. 13-20). Milwaukee: Marquette University Press.

Longuenesse, B. (2000). Kant and the Capacity to Judge. Princeton: Princeton University Press.

McNulty, M. B. (2014). Kant on Chemistry and the Application of Mathematics in Natural Science. Kantian Review, 19(3), 393418. https://doi.org/10.1017/S136941541400017X

McNulty, M. B. (2018). Kant on Empirical Psychology and Experimentation. En V. L. Waibel, M. Ruffing, \& D. Wagner (Eds.), Natur und Freiheit: Akten des XII. Internationalen KantKongresses (t. 4, pp. 2707-2714 ). Berlin: De Gruyter. https:// doi.org/10.1515/9783110467888-270

Melnick, A. (2006). Second Analogy. En G. Bird (Ed.), A Companion to Kant (pp. 169-181). Oxford/Malden: Blackwell Publishing.

Mischel, Th. (1967). Kant on the Possibility of a Science of Psychology. The Monist, 51(4), 599-622. https://www.jstor. org/stable/27902053

Nayak, A. C., \& Sotnak, E. (1995). Kant on the Impossibility of the "Soft Sciences". Philosophy and Phenomenological Research, 55(1), 133-151. https://www.jstor.org/stable/2108312 
Paton, H. J. (1970). Kant's Metaphysic of Experience. A Commentary on the First Half of the Kritik der reinen Vernunft. London: George Allen \& Unwin Ltd.

Plaass, P. (1994). Kant's Theory of Natural Science. Dordrecht: Springer.

Pérez, D. O. (2017). Los límites de la psicología como ciencia y la posibilidad de su uso en la antropología en Kant. Studia Kantiana, 15(2), 51-61. http://www.sociedadekant.org/studiakantiana/index.php/sk/article/view/298/260

Rosales, A. (1993). Una pregunta sobre el tiempo. En A. Rosales, Siete ensayos sobre Kant (pp. 225-250). Mérida: Universidad de los Andes.

Schönrich, G. (1991). Kant und die vermeintliche Unmöglichkeit einer wissenschaftlichen Psychologie. Psychologie und Geschichte, 2(3), 130-137. http://dx.doi.org/10.23668/psycharchives.584

Sturm, T. (2001). Kant on Empirical Psychology: How Not to Investigate the Human Mind. En E. Watkins (Ed.), Kant and the Sciences (pp. 163-184). Oxford: Oxford University Press.
Sturm, T. (2006). Is There a Problem with Mathematical Psychology in the Eighteenth Century? A Fresh Look at Kant's old Argument. Journal of the History of the Behavioral Sciences, 42(4), 353-377. https://doi.org/10.1002/jhbs.20191

Sturm, T., \& Wunderlich, F. (2010). Kant and the Scientific Study of Consciousness. History of the Human Sciences, 23(3), 48-71. https://doi.org/10.1177/0952695110363355

Teo, T. (2005). The Critique of Psychology. From Kant to Postcolonial Theories. New York: Springer.

Van Cleve, J. (1979). Substance, Matter, and Kant's First Analogy. Kant Studien, 70(2), 149-161. https://doi.org/10.1515/ kant.1979.70.1-4.149

Vigo, A. G. (2015). Kategoriale Synthesis und Einheit des Bewusstseins. Zu Kants Lehre vom Verhältnis zwischen Wahrnehmung und Erfahrung. En R. Enskat (Ed.), Kants Theorie der Erfahrung (pp. 169-199). Berlín: De Gruyter. 


\section{Notas}

1 Todas las referencias a textos de Kant diferentes de la Crítica de la razón pura son dadas de acuerdo con el tomo y la paginación de la edición académica de las obras de Kant (AA = Akademie-Ausgabe; Kant, 1900ss.). Las referencias a la primera Crítica, en cambio, son dadas según la paginación de la primera $(1781=\mathrm{A})$ y la segunda $(1787=\mathrm{B})$ ediciones originales. Las traducciones empleadas son las siguientes: para Anth (Anthropologie in pragmatischer Hinsicht / Antropología en sentido pragmático), la de Mario Caimi (Kant, 2009b); para KrV (Kritik der reinen Vernunft / Crítica de la razón pura), la de Mario Caimi (Kant, 2009a); y para MAN, las de Carlos Másmela (Kant, 1989) y Samuel Nemirovsky (Kant, 1993). (Estas dos últimas versiones de MAN fueron consideradas, con el texto alemán a la vista, para ofrecer una traducción única).

2 En Sobre la forma y principios del mundo sensible y del inteligible (1770; AA II, pp. 385ss.), Kant presenta una doctrina del espacio y del tiempo. La comparación de la misma con la doctrina correspondiente de la Crítica de la razón pura (1781/1787) excede los límites del presente trabajo.

3 Los estudios respecto de los que el nuestro ofrece el mencionado aporte son los siguientes: Frierson (2014), Gouaux (1972), Hatfield (1992), Leary (1982), McNulty (2018), Mischel (1967), Nayak y Sotnak (1995), Schönrich (1991), Sturm (2001 y 2006), Sturm y Wunderlich (2010) y Teo (2005). Cf. esp. infra, n. 15 y la tercera sección de nuestro artículo.

4 Los nombres completos de estas categorías son, respectivamente: "de inherencia y subsistencia (substantia et accidens)", "de causalidad y dependencia (causa y efecto)" y "de comunidad (acción recíproca entre el agente y el paciente"). (A80/B106).

5 Ciertamente, el espacio es forma del sentido externo, mientras que el tiempo es forma del sentido interno. Ahora bien, debido a que toda intuición, refiérase a fenómenos internos o externos, es una representación dada en el sentido interno, el tiempo es mediatamente forma de los fenómenos externos. Cf. A34/B50.

6 En Arias-Albisu (2011) exploramos, entre otros problemas, una dualidad en el concepto de substancia presentado por Kant en KrV. Por un lado, están los objetos relativamente permanentes de nuestra experiencia cotidiana, como sillas y piedras $\left(\right.$ substancias $\left._{1}\right)$. Por el otro, existe un elemento objetivo absolutamente permanente, esto es, la materia (substancia ${ }_{2}$ ). Las substancias $_{1}$ son substancias solamente en un sentido amplio del término. En primer lugar, las substancias, existen únicamente durante un cierto período. En segundo lugar, las substancias deben ser entendidas como accidentes o estados de la substancia ${ }_{2}$ que existe en todo tiempo. Este segundo señalamiento muestra que las dos concepciones de substancia que acabamos de exponer son partes de una única doctrina coherente. Igualmente, con el fin de simplificar, en este artículo consideramos únicamente el concepto de substancia $_{1}$. Nos referiremos a las substancias ${ }_{1}$ con la expresión "objetos substanciales". (Acerca de la permanencia de la substancia y la mutabilidad de sus accidentes, véase infra, n. 7). Debe notarse que, en $\mathrm{KrV}$, Kant presenta su concepción de substancia fundamentalmente en la "Primera analogía" (A182/B224ss.). Según A, el principio justificado en esa sección de KrV es: "[t]odos los fenómenos contienen lo permanente (substancia) como el objeto mismo, y lo mudable, como mera determinación de aquel, es decir, [como] un modo como el objeto existe" (A182). De acuerdo con B, tal principio es: "[e]n todo cambio de los fenómenos permanece la substancia, y el quantum de ella no se acrecienta ni disminuye en la naturaleza" (B224). Parece claro que el principio justificado en B se refiere solamente a la concepción de substancia ${ }_{2}$. Con el fin de fundamentar nuestra adscripción a Kant de la concepción de substancia ${ }_{1}$, disponemos de espacio en este artículo solamente para efectuar dos señalamientos. 1) En la "Tercera analogía", el término "Substanzen" (substancias) es utilizado como sinónimo de los términos "Dinge" (cosas) y "Gegenstände" (objetos). Asimismo, se hace referencia a la Tierra y a la Luna como casos particulares de substancias (B257). 2) En el último párrafo de la "Primera analogía" se asevera: "[a]sí, según esto, la permanencia es una condición necesaria, solo bajo la cual los fenómenos son determinables en una experiencia posible como cosas [Dinge] u objetos [Gegenstände]" (A189/B232). En el pasaje reproducido se sostiene que la determinación fenoménica correspondiente a la categoría de substancia (esto es, "la permanencia" y, agregamos nosotros, la mutabilidad a ella asociada) es una condición necesaria para que los fenómenos sean "determinables como cosas u objetos". Esta tesis puede entenderse en el sentido de que la aplicación de la categoría de substancia y la permanencia y la mutabilidad vinculadas con ella constituyen a las intuiciones empíricas como las cosas u objetos que son dados en nuestra experiencia cotidiana. Tales intuiciones empíricas se conforman así como objetos empíricos, en la medida en que ellos están en el espacio, son relativamente permanentes y están dotados de accidentes mudables. Además, es evidente que el término "cosas" (Dinge) no hace referencia en el pasaje citado a las cosas en sí mismas, es decir, consideradas con independencia de las formas de la sensibilidad humana, sino a los objetos empíricos. De hecho, en el texto reproducido se afirma que los fenómenos son determinables como cosas "en una experiencia posible". Véase Arias-Albisu (2011). No tenemos lugar para tratar el problema de si la permanencia y la mutabilidad fenoménicas son, al menos parcialmente, generadas de modo sintético mediante la aplicación de la categoría de substancia, o si son factores meramente dados a los que se aplica la categoría en cuestión. Señalemos, sin embargo, que consideramos correcta la primera de estas dos opciones. Para una argumentación a favor de la misma, véase Rosales (1993, pp. 236-239. Cf. Arias-Albisu, 2011 e infra, n. 7).

7 Para Kant, un accidente de una substancia no es en sentido estricto algo separable de ella. Más bien, por un lado, un accidente es un modo en el cual una substancia existe y, por el otro, una substancia no es más que lo que sus accidentes manifiestan de ella. Cf. A186-187/B229-230 y Longuenesse (2000, p. 330). Además, en A187/B230, "estado" (Zustand) es empleado como sinónimo de determinación o modo de existir de una substancia. Por tanto, también este término haría referencia a un accidente de una substancia. Acerca de este concepto kantiano de la substancia y sus accidentes y la relación del mismo con la concepción de la substancia de John Locke, cf. Longuenesse (2000, pp. 330-331). Véase también Arias-Albisu (2011).

8 En la "Segunda analogía", Kant se refiere al acontecimiento con el substantivo "Begebenheit" y el verbo "geschehen" (acontecer, suceder).

9 Sin embargo, notemos que Allison distingue entre los principios "todo-evento-alguna causa" y "misma-causa-mismo-evento". El primer principio establece que todo lo que comienza a existir tiene una causa de su existencia, y el segundo, que causas similares producen efectos similares. Según Allison, en la "Segunda analogía” de KrV se intentaría probar únicamente el principio 
"todo-evento-alguna-causa" (cf. Allison, 1992, pp. 335-336, 356-357; 2008, pp. 527, 541. Allison sigue en este punto a Beck, 1978, pp. 120, 126-127). Coincidimos con Longuenesse cuando afirma que no es posible disociar los dos principios mencionados en la "Segunda analogía". En efecto, Kant procura demostrar el principio "todo-evento-alguna-causa" probando, precisamente, que no es posible percibir un evento sin presuponer que sigue a algo en conformidad con una ley causal empírica ("misma-causa-mismo-efecto"). Cf. Longuenesse (2000, p. 369, n. 77). El examen de la validez de esta demostración no puede llevarse a cabo en este artículo por motivos de espacio. Por el mismo motivo, aquí podemos solamente presentar dos indicaciones a favor de la interpretación de Longuenesse. En primer lugar, la caracterización del esquema transcendental de la categoría de causalidad, que, en pocas palabras, es la expresión temporal y empírica de esta categoría, parece contradecir la interpretación de Beck y Allison. Tal esquema es "lo real, a lo cual, cuando el capricho manda que sea puesto, siempre le sigue algo diferente. Consiste, pues, en la sucesión de lo múltiple, en la medida en que está sometida a una regla" (A144/B183). Consideramos que "siempre" (jederzeit) hace referencia a que a todas las causas del tipo A les siguen efectos del tipo B. En segundo lugar, en la "Segunda analogía" se afirma: "[s]egún una regla tal, pues, en aquello que en general precede a un acontecimiento debe residir la condición para una regla según la cual siempre [jederzeit], y necesariamente, se sigue ese acontecimiento" (A193/B238-239). Como señala agudamente Longuenesse, el término "regla" (Regel) debe tener un sentido diferente en cada una de sus dos apariciones en esta oración. En su primera aparición, "regla" tiene el sentido de principio del entendimiento según el cual todo lo que acontece presupone algo diferente a lo cual sigue según una regla. Por otra parte, el término "regla", en su segunda aparición en el texto mencionado, tendría el sentido de regla objetiva empírica, según la cual el mismo tipo de efecto se sigue del mismo tipo de causa, esto es, el sentido de ley causal empírica (acerca de este último sentido de "regla", véase A200/B245. Para una explicación más completa de estas cuestiones, véase Longuenesse, 2000, pp. 368-370. En lo que hace a la expresión "condición para una regla", que está presente en el texto citado, el substantivo "condición" haría referencia al tipo de estado que oficia de causa). Esta breve interpretación del pasaje reproducido sugiere que en la "Segunda analogía" Kant tiene en cuenta tanto el principio"todo-evento-alguna causa" como el principio "misma-causa-mismo-evento" y que ellos están vinculados entre sí. Por último, notemos que también Melnick (2006) considera que Kant sostiene en la "Segunda analogía" los dos principios mencionados.

10 Asimismo, la aplicación de la categoría de comunidad presupone la aplicación de las categorías de substancia y de causalidad, y es una condición de la representación de relaciones de causalidad recíproca entre objetos substanciales. Nos limitamos a caracterizar la relación de acción o causalidad recíproca, cuyo estudio no es necesario para nuestros propósitos. Una substancia A sostiene una relación de causalidad recíproca con una substancia B si y solo si: 1) A posee al menos un accidente a1 que es causa de un cambio de accidentes $\beta 1$ en B, y 2) B posee al menos un accidente b1 que es causa de un cambio de accidentes $\alpha 1$ en A (cf. la discusión de Paton, 1970, t. II, pp. 294-331, quien examina distintas concepciones de la relación de acción o causalidad recíproca). Por ejemplo, la Tierra y la Luna sostienen una relación de causalidad recíproca. En efecto: 1) la fuerza atractiva de la Tierra es causa del movimiento de la Luna, y 2) la fuerza atractiva de la Luna es causa del movimiento periódico de los niveles de los mares en la Tierra (mareas). El ejemplo de la Tierra y la Luna, mencionado por Kant en la "Tercera analogía", fue presentado de acuerdo con indicaciones de Longuenesse (2000, pp. 385-386).

11 Es evidente que los exámenes observacionales requieren cuando menos la permanencia relativa de los objetos substanciales, y que la realización de experimentos presupone la posibilidad de conocer y manipular un objeto substancial. Como se señaló, la experiencia de un objeto substancial es posibilitada especialmente por la aplicación de la categoría de substancia, y esta aplicación no es posible en el ámbito de los fenómenos internos.

12 Es preciso destacar que en A33/B50 se emplea el término “analogía” para designar la representación del tiempo en términos de espacio.

13 Cf. A187/B230-231, Van Cleve (1979, p. 151) y Arias-Albisu (2011). Asimismo, al comienzo de la "Foronomía" de MAN, Kant afirma: "[c]omo en la foronomía no debe hablarse de ninguna otra cosa que de movimiento, no se atribuye aquí al sujeto del mismo, a saber, a la materia, ninguna otra propiedad que la movilidad. Por tanto, la materia misma puede ser válida también entretanto como un punto, y se abstrae en la foronomía de toda constitución interna (...)" (MAN, AA IV, p. 480). Debe notarse que, en su "Introducción y comentario de los traductores" a Plaass (1994, pp. 7-162), A. E. Miller y M. G. Miller sostienen: "[c]omo se vuelve importante en los capítulos posteriores, la conexión entre las varias determinaciones espaciales secuenciales que comprende un movimiento en un segmento de línea continuo es realmente posible solo porque están unidas como determinaciones de algún subyacente substrato persistente del movimiento, esto es, de algo "movible en el espacio"' (Plaass, 1994, p. 34). Estimamos que el señalamiento de A. E. y M. G. Miller permite afirmar, trasladándonos de MAN a KrV, que las categorías solo pueden conformar un acontecimiento en el sentido riguroso del término si se aplican constitutivamente a algo permanente y empírico dado en el espacio como substrato del acontecimiento.

14 De acuerdo con un pasaje de B152, las leyes que versan acerca de los fenómenos internos y son entonces estudiadas por la psicología empírica son las leyes empíricas de asociación de representaciones (cf. A100 y Anth, AA VII, p. 176). Un ejemplo de ley de asociación es la que vincula las representaciones de objetos empíricos experimentados sucesiva o simultáneamente en el tiempo. Un caso particular de esta ley es la asociación, en un individuo, entre, por un lado, las doce campanadas dadas por un reloj a los mediodías y, por el otro, los alimentos consumidos durante los almuerzos. Como estas dos clases de representaciones son usualmente sucesivas en el tiempo, se establece entre ellas un vínculo de asociación, de modo tal que las primeras provocan la aparición de las segundas en la mente del individuo en cuestión (cf. V-Anth/Fried, AA XXV, pp. 512-514. "V-Anth/Fried" designa la Antropología Friedländer, una colección de apuntes de lecciones de antropología dadas por Kant en el curso del semestre de invierno de 1775-1776). Por otro lado, en algunas Lecciones de Antropología, así como en la publicada Antropología en sentido pragmático (Anth), se encuentran exposiciones que sugieren que Kant también concibió leyes psicológicas diferentes de las mencionadas. Por ejemplo, Kant examina diferentes principios que rigen el aumento y la disminución del grado de intensidad de las sensaciones (Anth, AA VII, pp. 162-165). En caso de que estos principios sean leyes causales, una de estas sería la que establece una conexión entre, por un lado, el contraste que una representación produce respecto de otra y, por el otro, un aumento del grado 
de intensidad de la representación en cuestión. Por ejemplo, la presencia de un terreno bien cultivado dentro de un desierto arenoso vivifica la representación del primero en virtud del contraste (Anth, AA VII, p. 162). Acerca de las diferentes leyes psicológicas estudiadas por Kant, véase Sturm (2001, p. 170; 2006, p. 368), Sturm y Wunderlich (2010, p. 59) y Kraus (2018, pp. 83-84). Por último, debe notarse que la exploración de la significación que tiene la inclusión de la psicología empírica en el proyecto de una antropología en sentido pragmático queda fuera del alcance de este artículo. Cf. A849/B877. Acerca de este tema, véanse Hinske (1999), Blanc-Brudes (2011), Pérez (2017) y Kraus (2018). Respecto del estatus de estas leyes, véase infra, n. 15.

15 No hay consenso entre los comentadores acerca de esta cuestión. La lectura según la cual las leyes de la psicología empírica son leyes causales naturales del mismo tipo que las de la parte empírica de la física matemática (véase infra) es ofrecida por Hatfield (1992, pp. 201, 208 y 218-219. A favor de la posibilidad de aplicar en sentido estricto la categoría de causalidad a fenómenos psicológicos argumentan Sturm, 2001; 2006; y Sturm y Wunderlich, 2010). Mischel, por el contrario, argumenta que, como la categoría de causalidad no puede ser aplicada en sentido estricto a los fenómenos internos, las leyes que se refieren a estos fenómenos no pueden ser leyes causales en sentido riguroso. Tras citar el pasaje de B291-292 (véase supra), Mischel añade que podemos emplear la categoría de causalidad en un "significado analógico" para hacer comprensibles los fenómenos internos en términos de los fenómenos externos, de modo semejante a como, según Kant, podríamos emplear la categoría de causalidad para hacer comprensible la relación entre noúmenos y fenómenos (B431-432). Esta aplicación en sentido analógico no podría originar conocimiento en un sentido estricto del término. Por tanto, las leyes de la psicología empírica tendrían un carácter exclusivamente clasificatorio y descriptivo (Mischel, 1967, pp. 607-609. En contra de la posibilidad de aplicar en sentido riguroso la categoría de causalidad a los fenómenos internos argumentan Gouaux, 1972, pp. 239-241 y Nayak y Sotnak, 1995, pp. 148-149). Nuestra interpretación coincide, en general, con la de Mischel. Aunque Kant emplee ocasionalmente un lenguaje causal al referirse a fenómenos internos (cf. Anth, AA VII, p. 162), lecturas como la de Hatfield no pueden explicar la imposibilidad, señalada por Kant, de aplicar en sentido estricto las categorías de la relación a estos fenómenos. Esta imposibilidad torna necesaria la interpretación del mencionado lenguaje en un sentido amplio, tal como hace Mischel con su concepción del "significado analógico" del uso de la categoría de causalidad. Es importante señalar que en A147/B186 Kant parece emplear la expresión "significado lógico" en un sentido similar al que Mischel destaca en la expresión "significado analógico" de B431. Se debe notar, por último, que Mischel parece ocasionalmente confundir el examen, llevado a cabo en $\mathrm{KrV}$, de las condiciones de posibilidad de la experiencia y, por tanto, de los objetos de esta experiencia en general (A154-158/B193-197), con un examen de las condiciones de posibilidad de los objetos de la mecánica newtoniana: "[s]i los principios del entendimiento están diseñados para explicar cómo nuestro conocimiento de objetos newtonianos es posible, entonces ellos no encajarán con estados mentales, que no son objetos newtonianos, y habrá problemas cuando uno trate de establecer la posibilidad del conocimiento psicológico por medio de ellos" (1967, p. 607). Aquí consideramos, en cambio, que los aportes de $\mathrm{KrV}$ y MAN deben diferenciarse cuidadosamente. En esta sección nos enfocamos en el aporte de KrV. En la siguiente, nos ocuparemos del aporte de MAN y, entre otras cosas, resumiremos el estatus de la física y la química, con el fin de contribuir a una determinación, por contraste, del estatus de la psicología empírica.

16 Acerca de estas diferentes conformaciones del tiempo, véase Caimi (2012). Por ejemplo: "[1]a categoría de substancia sintetiza la multiplicidad del tiempo de modo tal que en él pueden distinguirse dos capas: una capa es la de las alteraciones cambiantes, otra capa es la de la permanencia. Es con referencia a la última que las alteraciones pueden ser percibidas. Así, el tiempo es determinado como un substrato perdurable, como el lecho de un rio sobre el cual fluye la corriente de eventos. Este estrato más profundo (la receptividad perdurable) se distingue de sus contenidos. Estos contenidos forman la segunda capa: la de los accidentes cambiantes. La serie del tiempo (...) llega a ser determinada ahora como una serie receptiva (...) capaz de soportar contenidos cambiantes" (p. 423). No tenemos espacio para examinar las modificaciones expuestas en el texto de Caimi de 2015 (pp. 213-217). Más adelante presentamos sintéticamente nuestra concepción del esquema de la categoría de substancia.

17 Mario Caimi traduce Zeitbestimmungen por "determinaciones del tiempo" (A145/B184; Kant, 2009, p. 199) e indica que esta expresión también se puede comprender como "determinaciones temporales". Estas últimas "no serían ya [...] determinaciones del tiempo mismo, sino que podrían ser, por ejemplo, determinaciones temporales de las cosas" (Kant, 2009, p. CXIII, n. 506)

18 Cf. Paton (1970, t. II, pp. 28-30), Allison (1992, pp. 286-287), Düsing (1995, p. 69) y Leppäkoski (1995, pp. 17ss.).

19 Agradecemos a un evaluador anónimo de Límite por haber formulado la objeción a la que intentamos responder y por recomendar la lectura de un artículo de Alejandro G. Vigo (2015). En este artículo Vigo sostiene que existen tres etapas en la teoría kantiana de la constitución de la objetividad. La primera corresponde a las categorías tomadas en sí mismas, esto es, sin referencia a objetos empíricos, sino únicamente en relación con objetos en general (2015, pp. 174-175). La segunda etapa refiere a la producción de los esquemas trascendentales mediante la imaginación productiva (2015, pp. 175-178). La tercera etapa corresponde a los juicios cognitivos y presupone no solo categorías esquematizadas, sino también conceptos empíricos. Tan solo en esta etapa hay una verdadera referencia a los objetos empíricos en sentido estricto (2015, pp. 178-180). Para nuestros fines, lo más relevante es que, según Vigo, los esquemas trascendentales son "determinaciones, realizadas a priori mediante las categorías, de la multiplicidad intuitiva contenida a priori en el tiempo" (2015, p. 176). Por tanto, Vigo parece sostener la interpretación de los esquemas trascendentales como determinaciones del tiempo presentada más arriba. Allí mostramos que esta lectura no es la única posible. Si bien no podemos desarrollar aquí este punto, es admisible señalar también que en nuestra inédita tesis doctoral, intitulada Significación y alcance de la doctrina kantiana del esquematismo trascendental (2010), hemos defendido una interpretación de, a grandes rasgos, las dos últimas etapas de la constitución de la objetividad distinguidas por Vigo como aspectos de una única aplicación de las categorías mediante sus esquemas.

20 Acerca de este punto, véanse Blomme (2011 y 2015), McNulty (2014) y los comentarios detallados de Plaass (1994) y Friedman (2013).

21 Durante la época de KrV (1781/1787) y MAN (1786), Kant adhiere a la química flogística desarrollada especialmente por Georg E. Stahl (Friedman, 1992, p. 265; véase Carrier, 2001 y Blomme, 2011 y 2015). Según Friedman (1992, p. 289), Kant se convirtió en seguidor de la química, de carácter antiflogístico, de Lavoisier, en 1795 como muy tarde. 
22 Resulta interesante señalar que Michael B. McNulty (2018) considera, erróneamente, que el que no sea posible realizar experimentos en el sentido interno no entraña principalmente que en el ámbito de la psicología empírica no sean posibles las leyes empíricas probables, sino que en esta disciplina no tienen cabida leyes empíricas estrictamente universales, esto es, que no admiten excepciones. Aquí consideramos, aunque no podamos desarrollar este punto, que una ley empírica, en tanto empírica, no puede alcanzar universalidad estricta, sino únicamente tornarse más probable en virtud de la efectuación exitosa de experimentos y observaciones rigurosas. Con certeza, McNulty no consigue justificar el tránsito de una ley empírica altamente probable a una ley empírica estrictamente universal. No es la falta de universalidad estricta la que afecta a las cuasileyes de la psicología empírica, sino la mencionada imposibilidad de adquirir probabilidad mediante experimentación y observación rigurosa. Respecto de la probabilidad de las leyes empíricas, entendidas como hipótesis, véase A647/B675, Lógica Jäsche (AA IX, pp. 84-86) y Arias-Albisu (2015).

23 Respecto de la observación rigurosa, es importante destacar que, en la Antropología en sentido pragmático, se afirma que en las experiencias externas "de objetos en el espacio (...) los objetos se presentan unos junto a otros, y se fijan como permanentes. El sentido interno ve las relaciones de sus determinaciones solo en el tiempo, y por tanto, en un fluir en el que la observación no es duradera" (Anth, AA VII, p. 134). Acerca de algunas dificultades para conocer, en el marco de la mencionada antropología pragmática, a los seres humanos, véase Anth (AA VII, pp. 120-121).

24 Es posible señalar que los historiadores de la psicología presentan diferentes evaluaciones de la influencia de la concepción kantiana de la psicología empírica en los pensadores posteriores. Por un lado, se ha señalado que esta influencia fue mayormente negativa, ya que consistió fundamentalmente en un estímulo para la investigación en contra de las críticas de Kant a la psicología empírica y la psicología racional (Teo, 2005, p. 42. La psicología racional es presentada críticamente por Kant en el capítulo "De los paralogismos de la razón pura" de KrV - A341ss./B399ss.- Según Kant, el objetivo de esta pretendida ciencia es obtener una totalidad de conocimiento acerca del sujeto pensante. Tal conocimiento debería alcanzarse, independientemente de la experiencia, por medio de inferencias que toman como base la sola proposición "yo pienso", esto es, el principio de la apercepción -B131ss.-). Por otro lado, se ha acentuado la influencia positiva de la concepción kantiana de la psicología empírica, y de la filosofía crítica en general, en pensadores subsiguientes que se ocuparon de cuestiones psicológicas (Leary, 1982, pp. 36-37. Sin embargo, véase p. 35).

25 La ausencia de una consideración de los pasajes mencionados se pone en evidencia más pronunciadamente en la discusión de Frierson acerca de si, para Kant, se encuentra cierta substancialidad en el sentido interno (2014: 21-26). Tras evaluar otros textos de $\mathrm{KrV}$ y aportes de algunos comentaristas, Frierson concluye: "[a] fin de cuentas, Kant no se define con respecto a la cuestión estrictamente metafísica de si el alma es una substancia. Pero tiene en claro que hay alguna substancia que subyace a los cambios observados en el sentido interno, y sea cual fuese la substancia que resulte ser, ella es suficiente para justificar la adscripción de leyes causales a esos estados psicológicos" (2014, p. 26. Cf. A379, citado en Frierson, 2014, p. 24). Es evidente que la discusión de Frierson se habría enriquecido si este autor hubiese tenido en cuenta la imposibilidad, señalada principalmente en los pasajes de $\mathrm{KrV}$ a los que hemos remitido, de constituir en el sentido interno una substancia fenoménica idéntica a las constituidas en el sentido externo. Es sustancial señalar que Frierson, inmediatamente antes de la conclusión que hemos reproducido, cita un pasaje del "Apéndice a la dialéctica transcendental" de KrV (esto es, A672/B700) en el que Kant presenta la función regulativa de la idea de alma. Sin embargo, Frierson no explica esta concepción. Como veremos a continuación, esa función regulativa desempeña un papel central en la interpretación de Kraus (2018).

26 Traducimos los pasajes de Kant citados por Kraus a partir de la versión inglesa que ella emplea (Kant, 1998). 